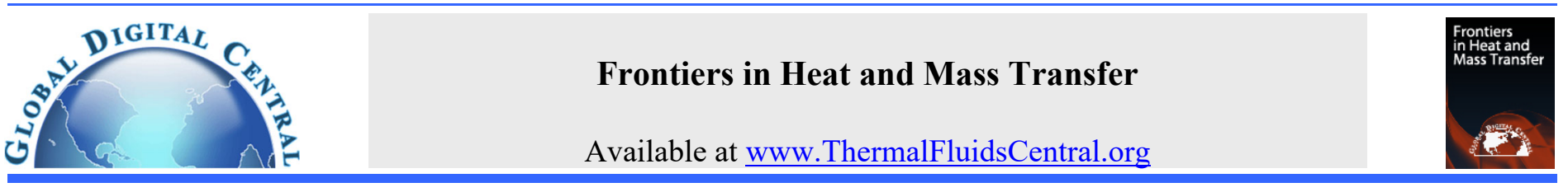

\title{
PHYSICAL HEAT TRANSFER
}

\author{
Hai-Dong Wang ${ }^{\mathrm{a}}$, Zeng-Yuan Guo ${ }^{\mathrm{a},{ }^{*}}$ \\ ${ }^{a}$ Department of Engineering Mechanics, Tsinghua University, Beijing 100084, People's Republic of China
}

\begin{abstract}
The classical heat transfer theory is established on the empirical models of Fourier's heat conduction law and Newton's cooling law. Although the classical theory has been successfully used in a wide range of industrial engineering applications, it lacks deep understanding of the physical mechanisms for energy transport and analytical methodology based on solid mathematical and mechanical principles. The rapid development of modern science and technology challenges the traditional heat transfer theory in two aspects: (1) Fourier's law of heat conduction is no longer valid under the ultra-fast laser heating or nanoscale conditions; (2) The optimization principle minimizing entropy generation is not suitable for heat transfer problems without heat to work conversion. In order to solve the first challenge, we re-examined the nature of heat and introduced new physical quantities, such as thermomass, thermomass velocity, thermomass energy. Then the heat transfer problems can be analyzed in the framework of vectorial fluid mechanics, and the thermomass momentum conservation equation has been established and known as a general law of heat transfer. For the second challenge, we introduced new physical quantities, entransy and entransy dissipation, by analogy between the heat conduction process and the electric conduction process. Unlike entropy as the core physical quantity in thermodynamics dealing with energy conversion, entransy is the quantity to represent the ability of heat transfer in an incompressible system. The entransy dissipation represents the irreversibility of heat transfer process. On this basis, the least action principle to minimize entransy dissipation can be used to optimize the heat transfer process and enhance energy efficiency. Because the physical essence of entransy is the thermomass potential energy, the entransy-based variational method is actually the energy method in heat transfer. All the new physical quantities, principles and methods form a new and independent knowledge system in heat transfer, which may be named "Physical heat transfer".
\end{abstract}

Keywords: Thermomass, general heat conduction law, entransy, energy efficiency.

\section{INTRODUCTION}

The classical heat transfer theory describes the rule of diffusive transport of thermal energy and sets the foundation for a wide range of industrial applications related to power engineering, thermal management, energy harvesting and conservation, etc (Lienhard, 2019). However, when we re-consider the theoretical foundation of classical heat transfer, it is known that except for the heat radiation theory, most principles and models in heat transfer are built on the empirical formulas like Fourier's heat conduction law and Newton's cooling law (Lienhard, 2019). The strict physical and mathematical analysis methods are lacking in the classical principles of heat transfer. This problem originates from the unique feature of heat transfer phenomena, as Fourier's statement in 1822 of that the effects of heat make up a special order of phenomena, which cannot be explained by the principles of motion and equilibrium ...... connected with dynamical theories (Kroes and Bakker, 2013). In 1980s some monographs were published in the field of thermal science, such as "Thermofluid mechanics", "Thermofluid dynamics", "Dynamics of heat" (Look Jr. et al., 1988; Reynolds, 1972; Fuchs, 2010), etc. They do have the terms "mechanics" or "dynamics" in the title, however, they are not using real mechanical or dynamical principles of heat in the analysis. Thermofluid mechanics or thermofluid dynamics is a theory that simply combines the disciplines of thermodynamics and fluid mechanics, each of them has principles on its own. This theory has been used in formulating and solving problems in reacting flows, plasma dynamics, compressible gas dynamics, etc., where the solution of fluid is coupled with complex heat and mass transfer problems. "Dynamics of heat" is developed in continuum thermodynamics to combine the classical theory of thermodynamics and the theory of heat transfer into one piece (Fuchs, 2010), but apparently, it doesn't bring up any new principles. In conclusion, the existed theories of thermofluid mechanics and dynamics of heat still follow the traditional analysis methods and the basic laws of thermodynamics and heat transfer. They are not built on real mechanical or dynamical principles of heat.

The rapid development of modern science and technology poses two challenges to the traditional theory of heat transfer, such as: (1) the classical Fourier's heat conduction law is no longer valid under the ultrafast laser heating and nanoscale conditions (Wang et al., 2012; Bishri, 1999; Herwig and Beckert, 2000; Zhou et al., 2008; Mohajer et al., 2016; Guo and Xu, 1992; Shiomi and Maruyama, 2006; Zhang et al., 2006); (2) thermodynamic optimization method based on minimum entropy generation principle is not suitable for heat transfer problems without heat to work conversion (Bertola and Cafaro, 2008; Kjelstrup et al., 2000; Prigogine, 1967; Bejan, 1977; Bejan, 1979; Bejan, 1982; Bejan, 1996; Bejan, 1996; Bejan, 2002; Hesselgreaves, 2000; Pandey and Nema, 2011; Sekulic, 1986). In order to solve the first challenge, unlike the existing approaches to meet the first challenge, they all revise the existing Fourier's law of heat conduction (Tzou, 1996; Rukolaine, 2014; Chen et al., 2006; Cattaneo, 1948; Vernotte, 1958). We take a re-examination of the nature of heat as a starting point, and then introduce new physical quantities such as thermomass, thermomass velocity and thermomass energy, so that the heat transfer process can be described by means of hydrodynamics and a general law of heat conduction has been 
established (Wang, 2014; Wang et al., 2010; Cao and Guo, 2007; Guo and Hou, 2010; Dong and Guo, 2011; Dong et al., 2017; Dong et al., 2012; Gupta and Mukhopadhyay, 2019; Freitas et al., 2016; Wang et al., 2014; Jou et al., 2011; Cimmelli et al., 2016; Dong, 2016). For the second challenge, the fundamental reason why the principle of minimum entropy generation is not applicable to the optimization of heat transfer without energy conversion is that entropy is the core physical quantity in thermodynamics, whose research object is the conversion law between heat and other forms of energy, rather than in heat transfer, whose research object is the transfer law of heat. Therefore, it is necessary to introduce some new physical quantities to describe the heat transfer process and its irreversibility. New physical quantities, such as entransy, entransy dissipation and entransy dissipation-based thermal resistance are introduced by analogy between the heat conduction process and the electric conduction process. On this basis, the principle of least action in heat transfer and the principle of minimum entransy dissipation-based thermal resistance for the optimization of heat transfer process are proposed (Chen et al., 2013; Cheng et al., 2012; Wang et al., 2017; Guo et al., 2003; Meng et al., 2005; Chen et al., 2008; Chen et al., 2011; Chen and Guo, 2010; Chen et al., 2009; Li and Chen, 2012; Song and Guo, 2011; Chen et al., 2007; Chen and Ren, 2008, Yuan and Chen, 2011; Jia et al., 2012; Cheng and Liang, 2011; Cheng et al., 2011; Liu et al., 2009). Finally, an energy method (similar to that in analytical mechanics) in heat transfer is established for energy efficiency optimization of thermal systems (Cheng and Liang, 2011; Xia et al., 2017). Since the new physical quantity, entransy, in fact is the simplification of thermomass potential energy, so the entransy dissipation is essentially the dissipation of thermomass potential energy in the process of heat transfer (Guo, 2018).

Based on the existing heat transfer theory, a series of new physical quantities are introduced, some new principles and laws are put forward by means of physical and mechanical principles, and new analytical methods are established, thus forming a new and independent knowledge system. Since the emphasis of the discussion is on the analysis of heat transfer law and its physical mechanism, rather than on their detailed mathematical processing, this knowledge system may be named as "physical heat transfer".

\section{NEW PHYSICAL QUANTITIES IN HEAT TRANSFER}

\subsection{Thermomass}

Einstein's theory of special relativity is well known for giving the formula between mass and energy as (Einstein, 1988):

$$
E=M c^{2}=\frac{M_{0} c^{2}}{\sqrt{1-u^{2} / c^{2}}} \approx M_{0} c^{2}+\frac{1}{2} M_{0} u^{2}, \quad \text { if } u<<c
$$

where $E, M$ and $c$ are the total energy, relativistic mass and speed of light, respectively. $M_{0}$ and $u$ are the rest mass and velocity of the object, respectively. The total energy of an object equals to the sum of its energy of rest mass, $M_{0} c^{2}$, and its kinetic energy, $1 / 2 M_{0} u^{2}$, when the velocity is much smaller than the speed of light. Considering an object of rest mass $M_{0}$ at a certain temperature, the thermal energy of the object due to thermal vibration of all the atoms is $E_{\mathrm{D} 0}$. If the velocity of object is zero, its kinetic energy is zero too, but the thermal energy cannot be neglected. Hence, the mass of a hot object is always larger than the mass when it is cold. The increased mass due to thermal vibration can be expressed as:

$$
M_{h}=\frac{E_{D 0}}{c^{2}}
$$

where the relativistic mass $M_{h}$ is named as "thermomass", i.e. the equivalent mass of thermal vibration of atoms (or phonon gas), proposed by Guo et al (Jia et al., 2012; Guo, 2018; Dong et al., 2011; Dong, 2015). It is clear that the total mass of an object equals to the sum of its rest mass and thermomass, and the absolute value of thermomass is usually very small comparing with the rest mass. However, as it will be discussed later, the inertia effect caused by the thermomass cannot be neglected under the extreme conditions of heat conduction, such as ultra-high heat flux and ultra-fast laser heating. Also, the concept of thermomass is the core of physical heat transfer theory, based on which, a series of new heat transfer quantities and some new laws or principles can be established. It is worth noting that the concept of thermomass is different from the concept of "caloric" in the 18th century (Mares et al., 2008; Fox, 1971). The caloric theory was an obsolete theory that heat consists of an imaginary, massless caloric fluid that flows from hotter bodies to colder bodies, while the thermomass of an object is the equivalent mass of its thermal energy based on Einstein's theory of relativity.

\subsection{Pressure, velocity of thermomass gas and thermomass energy}

A normal gas consists of a large number of randomly moving molecules. Following the same definition of thermomass, a particle that only has moving mass, that is, the relativistic mass of each molecule can be defined as "Thermon", while its rest mass equals zero (Wang and Guo, 2010). It is natural to think of a thermomass gas consisting of a large number of thermons. The following microscale picture of heat transfer can be understood as the directional flow of thermomass gas from high temperature to low temperature.

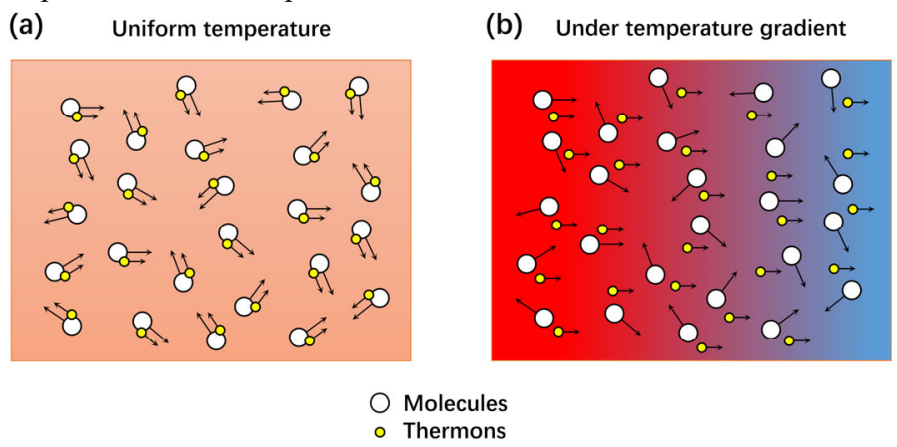

Fig. 1 Illustration of moving thermons at uniform temperature (a) or under temperature gradient (b).

Figure 1 shows the illustration of moving thermons in solid. Fig. 1(a) is the case of uniform temperature, the thermons attached to the molecules are randomly moving and the net flow is zero. Fig. 1(b) is the case under temperature gradient, the thermons are driven to flow from high temperature to low temperature and form a heat flow. The pressure gradient of thermomass gas is the driving force of thermons. In dielectric solids, the thermomass gas is actually phonon gas, since phonons are the energy carriers with relativistic masses. The state equation of phonon gas can be derived from the Debye state equation of solids, written as:

$$
P=-\frac{\partial E}{\partial V}+\gamma \frac{\partial E_{D 0}}{V}
$$

where $P$ is the pressure, $V$ is the volume, $\gamma$ is the Grüneisen constant. The first term states the interactions between atoms in solids, and the second term states the contribution of lattice vibration at non-zero temperature. The pressure of phonon gas due to thermal vibration is the pressure of thermomass gas, defined as $P_{h}$ written as:

$$
P_{h}=\gamma \frac{\partial E_{D 0}}{V}=\gamma \rho_{h} C T=\frac{\gamma \rho}{c^{2}}(C T)^{2}
$$

where $\rho_{h}=\rho C T / c^{2}$ is the density of thermomass gas, $C, \rho$ and $T$ are the capacity, density and temperature of solid, respectively. Equation (4) is the state equation of the thermomass gas. It is seen that the pressure of thermomass gas is proportional to the square of temperature, different from the ideal gas, in which the pressure is proportional to temperature at constant volume. The pressure of thermomass gas in solid is usually very small. For example, $P_{h}$ is only $5 \times 10^{-3} \mathrm{~Pa}$ in silicon at room temperature. Even though, the pressure gradient of $P_{h}$ is the driving force of thermomass gas, i.e. the driving force of heat flow in solid. The firstorder derivative of $P_{h}$ in space is proportional to the temperature gradient, 
which is consistent with the well-known fact that the temperature gradient is the driving force of heat flow.

In the existing heat transfer theory, there is only physical quantity of heat flow or heat flux density, but no physical quantity of heat transfer velocity. Based on the concept of thermomass, the quantitative relationship between heat transfer velocity and heat flux can be extracted:

$$
\frac{q}{c^{2}}=\rho_{h} u_{h}=\frac{\rho C T}{c^{2}} u_{h}, \rho_{h}=\rho C T / c^{2}, q=\rho C T u_{h},
$$

where $u_{h}$ is the heat transfer velocity, that is, the drift velocity of thermomass gas. The mass flow rate of thermomass gas can be calculated as $\dot{m}_{h}=\rho h u_{h}=q / c^{2}$. In dielectrics, the velocity of thermomass gas is the macroscopic velocity of phonon gas. Therefore, the kinetic and potential energy of per unit thermomass is $0.5 \rho h u h^{2}$ and $0.5 \rho h C T$ respectively (Cao and Guo, 2007). Here, we would like to note that the thermomass potential energy is a new form of energy, that is, the relativistic energy.

\subsection{Entransy and entransy dissipation}

The analogies among electrical conduction, fluid flow, and heat conduction listed in Table 1 were used to introduce a new physical quantity, where the temperature, as the thermal potential, corresponds to the electrical potential or the gravitation potential, the thermal energy stored in an incompressible object corresponds to the electrical charge stored in a capacitor or the mass of a fluid in a vessel, and Fourier's law corresponds to Ohm's law or Darcy's law. However, there is no quantity in heat transfer theory corresponding to the electrical potential energy of a capacitor or the gravitation potential energy of a fluid in a vessel. Hence, Guo et al. (Chen et al., 2013; Xu, 2011) defined the missing quantity, $G$, with the differential form:

$$
d G=U d T=M C T d T=V \rho C d T
$$

and its integral form:

$$
G=U T / 2=M C T^{2} / 2
$$

where $C$ is the constant volume specific heat capacity, $T$ is the temperature, $M$ is the mass, $U$ is the internal energy of an incompressible object and $G$ is a state quantity called entransy, which represents the heat transfer ability of an object. Dividing the entransy by the volume gives the entransy density:

$$
g=G / V=\rho C T^{2} / 2
$$

\begin{tabular}{|c|c|c|c|c|}
\hline & $\begin{array}{c}\text { Non- } \\
\text { dissipative } \\
\text { quantity }\end{array}$ & Potential & $\begin{array}{c}\text { Dissipative } \\
\text { quantity }\end{array}$ & Transport law \\
\hline \multirow{2}{*}{$\begin{array}{l}\text { Electro- } \\
\text { nics }\end{array}$} & $\begin{array}{l}\text { Electric } \\
\text { charge }\end{array}$ & $\begin{array}{c}\text { Electric } \\
\text { potential }\end{array}$ & $\begin{array}{c}\text { Electric } \\
\text { potential } \\
\text { energy }\end{array}$ & Ohm's law \\
\hline & $Q_{e}$ & $V$ & $E_{e}=Q V / 2$ & $i=-\sigma \frac{\mathrm{d} v}{\mathrm{~d} x}$ \\
\hline \multirow{2}{*}{$\begin{array}{l}\text { Fluid } \\
\text { mechan- } \\
\text { ics }\end{array}$} & Mass & $\begin{array}{l}\text { Gravitation } \\
\text { al potential }\end{array}$ & $\begin{array}{c}\text { Gravitation } \\
\text { al potential } \\
\text { energy }\end{array}$ & Darcy's law \\
\hline & $M$ & $H g$ & $\begin{array}{c}E_{m}= \\
M H g / 2\end{array}$ & $\dot{m}=-\frac{k A}{\mu} \frac{\mathrm{d} p}{\mathrm{~d} y}$ \\
\hline \multirow{2}{*}{$\begin{array}{l}\text { Heat } \\
\text { transfer }\end{array}$} & Heat & $\begin{array}{l}\text { Thermal } \\
\text { potential }\end{array}$ & $\begin{array}{c}\text { Thermal } \\
\text { potential } \\
\text { energy }\end{array}$ & Fourier's law \\
\hline & $Q_{h}$ & $T$ & $\begin{array}{c}E_{h}= \\
C\left(M C T^{2}\right) / 2 \\
c^{2}\end{array}$ & $q=-\lambda \frac{\mathrm{d} T}{\mathrm{~d} x}$ \\
\hline
\end{tabular}

where $\rho$ is the density and $V$ is the object volume.

Table 1 Analogies among heat conduction, fluid flow and electrical conduction (Guo et al., 2007)
Comparing $E_{h}$ with $G$ in Eq. (7) shows that these two quantities are the same except for a constant $C / c^{2}$. It is saying that the entransy is actually a simplified expression of thermomass potential energy. Therefore, the theory of entransy can be unified with the theory of thermomass.

If one multiplies both sides of energy conservation equation without internal heat source by temperature, the entransy balance equation can be obtained as (Chen et al., 2013; Guo et al., 2007):

$$
\frac{1}{2} \frac{\partial\left(\rho C T^{2}\right)}{\partial t}=-\nabla(q T)+q \nabla T
$$

The left side of equal sign is the time variation of entransy density, $g=$ $\rho C T^{2} / 2$, two terms on the right side of equal sign are the entransy flux and local entransy dissipation rate, respectively. Assuming that two bodies $\mathrm{A}$ and $\mathrm{B}$ with different initial temperatures $T_{A}$ and $T_{B}$ are in contact with each other and then reach a same temperature. Before contact, the entransy of system is $M_{A} C_{A} T_{A}^{2} / 2+M_{B} C_{B} T_{B}^{2} / 2$, after contact, the total entransy changes to $\left(M_{A} C_{A}+M_{B} C_{B}\right) / T_{A B}{ }^{2} / 2$, where $T_{A B}=\left(M_{A} C_{A} T_{A}\right.$ $\left.+M_{B} C_{B} T_{B}\right) /\left(M_{A} C_{A}+M_{B} C_{B}\right)$. After a simple calculation, it is known that the entransy of system is reduced after reaching the equilibrium temperature $T_{A B}$ due to the entransy dissipation caused by the heat conduction between A and B (Chen et al., 2013). Cheng has proved that the total entransy always decreases for an isolated system without internal heat generation (Cheng et al., 2011). All these indicate that the entransy dissipation is a measure of irreversibility of heat transfer without energy conversion. In view of the fact that the entransy is a simplified expression of thermomass potential energy, the entransy dissipation is actually the thermomass energy dissipation during heat transfer.

Entropy and entransy are two important parameters in thermal science. Thus, the differences between entransy theory and entropy theory for heat transfer analysis and optimization are related to the definition and physical meaning of the state quantity, heat transfer purpose, the optimization objective, the optimization principle and the optimization criterion, as listed in Table 2.

Table 2 Entropy theory and entransy theory (Chen et al., 2011; Chen et al., 2009)

\begin{tabular}{|c|c|c|}
\hline Research objective & $\begin{array}{c}\text { Entropy theory } \\
\text { Wonversion of heat to } \\
\text { work }\end{array}$ & $\begin{array}{c}\text { Entransy theory } \\
\text { Object heating or } \\
\text { cooling }\end{array}$ \\
\hline $\begin{array}{c}\text { Definition of state } \\
\text { quantity and its } \\
\text { physical meaning }\end{array}$ & $\begin{array}{c}\text { Entropy: } S=S(T, V) \\
\text { Conversion ability of } \\
\text { heat to work }\end{array}$ & $\begin{array}{c}\text { Entransy: } G=G(T) \\
\text { Transfer ability of } \\
\text { heat }\end{array}$ \\
\hline $\begin{array}{c}\text { Irreversibility of } \\
\text { thermal process }\end{array}$ & $\begin{array}{c}\text { Entropy generation } \\
\text { rate: }\end{array}$ & $\begin{array}{c}\text { Entransy dissipation } \\
\text { rate: }\end{array}$ \\
\hline $\begin{array}{c}S_{g}=\frac{\lambda}{T^{2}}\left(\frac{\mathrm{d} T}{\mathrm{~d} x}\right)^{2} \\
\text { objimization }\end{array}$ & $\begin{array}{c}\text { Maximum conversion } \\
\text { efficiency of heat to } \\
\text { work }\end{array}$ & $\begin{array}{c}\text { Maximum heat } \\
\text { transfer efficiency }\end{array}$ \\
\hline $\begin{array}{c}\text { Optimization } \\
\text { principle }\end{array}$ & $\left.\begin{array}{c}\text { Minimum entropy } \\
\text { generation }\end{array}\right)^{2}$ & $\begin{array}{c}\text { Minimum entransy } \\
\text { dissipation-based } \\
\text { thermal resistance }\end{array}$ \\
\hline $\begin{array}{c}\text { Optimization } \\
\text { criterion }\end{array}$ & None & $\begin{array}{c}\text { Uniformity of } \\
\text { temperature gradient } \\
\text { for heat conduction; } \\
\text { field synergy degree } \\
\text { for heat convection }\end{array}$ \\
\hline
\end{tabular}

It is seen that entropy and entransy are both state quantities and they represent a certain irreversibility of the process. Entropy is the parameter to show the ability of heat to work conversion, while entransy is the parameter to show the ability of heat transfer. Entropy generation rate can be used to optimize the thermodynamics process, while entransy 
dissipation rate can be used to optimize the heat transfer process. The difference between entransy dissipation rate and entransy dissipation function is the minimum action (Lagrange's function) of the heat transfer process. The entransy dissipation is the measure of heat transfer irreversibility without heat to work conversion. Both entropy and entransy can be applied in thermal optimization, but they do have some differences in the real applications. The minimum entropy generation principle has been used in the optimization of heat exchangers, but the optimum result is not always corresponding to the minimum entropy generation. On the other hand, the minimum entransy dissipation principle does not have such problem. The optimum structure of heat exchanger is corresponding to the minimum entransy dissipation.

\section{NEW LAWS AND NEW PRINCIPLES IN HEAT TRANSFER}

\subsection{General heat conduction law}

Considering the heat conduction in solid without internal heat source, the continuity and momentum equations of thermomass gas are given as:

$$
\begin{gathered}
\frac{\partial \rho_{h}}{\partial t}+\nabla \cdot\left(\rho_{h} u_{h}\right)=0 \\
\rho_{h} \frac{\mathrm{D}}{\mathrm{D} t}\left(\rho_{h} u_{h}\right)+\nabla P_{h}+f_{h}=0
\end{gathered}
$$

The governing Eqs. (10) and (11) of thermomass gas are written according to the principles of fluid mechanics, and Eq. (10) is actually the thermal energy conservation equation. Three terms on the left side of Eq. (11) are the inertia force, driving force and resistance of thermomass gas. To be more specific, in $1 \mathrm{D}$ heat conduction problem, the steady state form of conservation equation of momentum, Eq. (11), can be written as:

$$
\rho_{h} u_{h} \frac{\mathrm{d} u_{h}}{\mathrm{~d} t}+\frac{\mathrm{d} P_{h}}{\mathrm{~d} x}+f_{h}=0
$$

Hence, heat has dual nature of energy and mass, that is, when heat is converted to other forms of energy, it acts as energy, and when heat is transferred, it acts as mass.

Heat conduction is actually the flow of thermomass gas in a porous medium, where the solid lattice forms the skeleton of porous medium. By using Darcy's law describing the linear relationship between flow velocity and resistance in porous medium (Cao and Guo, 2007), the resistance of thermomass can be given as $f_{h}=\beta_{h} u_{h}$, where $\beta_{h}=2 \gamma c^{2} \rho h^{2} C / \lambda$ is the linear coefficient. A complete expression of $1 \mathrm{D}$ general heat conduction law is:

$$
\tau_{h}\left(\frac{\partial q}{\partial t}-\frac{q}{T} \frac{\partial T}{\partial t}+\frac{q}{\rho C T} \frac{\partial q}{\partial x}-\frac{q^{2}}{\rho C T^{2}} \frac{\partial T}{\partial x}\right)+\lambda \frac{\partial T}{\partial x}+q=0
$$

where $\lambda$ is the thermal conductivity and $\tau_{h}=\lambda /\left(2 \gamma \rho C^{2} T\right)$ is the relaxation time of thermomass gas. If the spatial inertia term is neglected, Eq. (13) returns to the Cattaneo-Vernotte $(\mathrm{C}-\mathrm{V})$ model describing the propagation of thermal wave (Maillet, 2019):

$$
\tau_{h} \frac{\partial q}{\partial t}+\lambda \frac{\partial T}{\partial x}+q=0
$$

If the temporal inertia term is neglected, Eq. (13) is written as:

$$
\lambda\left(1-\frac{q^{2}}{2 \gamma \rho^{2} C^{3} T^{3}}\right) \frac{\mathrm{d} T}{\mathrm{~d} x}+q=0
$$

Equation (15) is the 1D general heat conduction law in steady states, where the term in brackets is the spatial inertia term of themomass. It demonstrates theoretically that non-Fourier heat conduction exists in steady states, as well as the thermal wave phenomena in transient states. The non-Fourier heat conduction originates from the non-negligible spatial inertia force, which plays an important role under the high heat flux or nanoscale conditions (Wang, 2014).

If the terms of inertia force in Eq. (13) is neglected, the momentum equation of thermomass gas is simplified as:

$$
\lambda \frac{\mathrm{d} T}{\mathrm{~d} x}+q=0
$$

Eq. (16) is the classical Fourier's law, which demonstrates that Fourier's law is the special case of the general heat conduction law when ignoring the inertia force. The physical explanation of Fourier's law based on thermomass theory is the balance equation between the driving force and resistant force.

Although the fluid mechanics has been well applied to describe the motion of thermomass gas, there are still several notable differences between thermomass gas and normal gas: (1) Thermomass is a fluid with relativistic mass, differing from the normal gas. This is the most essential difference between these two kinds of fluids. Thermomass has zero static mass, but the static mass of real fluid is not zero. (2) There have to be two independent parameters in the state equation of normal gas, e.g. temperature and pressure. However, for the thermomass gas, temperature is the only independent parameter in the state equation. Once the temperature of system is fixed, the internal energy $M C T$ and the thermomass $M C T / c^{2}$ are fixed as well. Also the quantity of thermomass is significantly smaller than that of real fluid. So the influence caused by thermomass is rather small under the normal conditions where the speed is much smaller than the light speed and the level of energy density is not very high. The inertia effect of thrmomass can be only detected when the heat flux is higher than $10^{10} \mathrm{~W} / \mathrm{m}^{2}$ as shown in the next experimental section. The unique characteristics of thermomass gas result in its distinctive dynamic behaviors.

\subsection{Experimental evidence for the spatial inertia of thermomass}

Equation (15) indicates that the non-Fourier heat conduction induced by spatial inertia of thermomass occurs under the high heat flux and low temperature conditions. It is quite difficult to reach an ultra-high heat flux level in bulk materials, but the heat flux in nanowire or film can be very high due to its ultra-small cross sectional area. Wang et al. experimentally studied the non-Fourier heat conduction in gold nanofilms with a heat flux over $10^{10} \mathrm{Wm}^{-2}$ at an ambient temperature down to $3 \mathrm{~K}$ (Wang et al., 2012). The gold nanofilm is about $10 \mu \mathrm{m}$ long, $300 \mathrm{~nm}$ wide and $76 \mathrm{~nm}$ thick, made by standard electron beam lithography and physical vapor deposition method. The temperature dependent electrical and thermal conductivities of nanofilm have been measured by using a self-heating method and used as known parameters in thermal analysis afterwards. In the experiment, the gold nanofilm was used as Joule heater and precise resistance thermometer at the same time. The curve of average temperature vs. heating power is shown in Fig. 2.
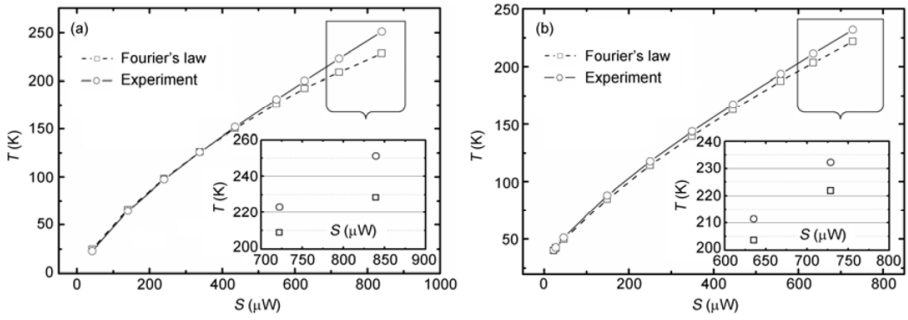

Fig. 2 Temperature vs. heating power of gold nanofilm: (a) the ambient temperature is $3 \mathrm{~K}$; (b) the ambient temperature is $30 \mathrm{~K}$. Reprinted with permissions from Springer Publishing Group (Wang et al., 2012).

Figure 2 (a) and (b) show the measured temperatures of gold nanofilm at different ambient temperatures, where the circles with solid line are the experimental data and the squares with dashed line are the calculated result from Fourier's law. It is seen that the experimental data agrees with the prediction of Fourier's law at low heating power, while a significant deviation occurs at high heating power (the maximum heat flux reaches $1.83 \times 10^{10} \mathrm{Wm}^{-2}$ ). Such deviation from Fourier's law is caused by the spatial inertia of thermomass. The temperature difference 
caused by non-Fourier heat conduction increases as the ambient temperature decreases, just like the general heat conduction law predicted.

\subsection{Applications of the general heat conduction law}

As mentioned above, the general heat conduction law can be used to study the non-Fourier heat conduction when the thermal inertia effect cannot be neglected. It has been successfully applied in studying the intrinsic thermal conductivity of nanostructure, thermal rectification, thermal wave propagation, etc. Some important applications are discussed here.

\subsubsection{Intrinsic thermal conductivity of carbon nanotube}

Equation (15) can be re-written as:

$$
\lambda_{\text {app }}=-\frac{q}{\mathrm{~d} T / \mathrm{d} x}=\lambda\left(1-\frac{q^{2}}{2 \gamma \rho^{2} C^{3} T^{3}}\right)
$$

where $\lambda_{\text {app }}$ is calculated as the ratio between heat flux and temperature gradient. This is the apparent thermal conductivity including influences caused by spatial inertia of thermomass. In Eq. (17), $\lambda$ on the left side of equal sign is the intrinsic thermal conductivity, which is independent of heat flux. If the data from experiment or simulation were analyzed by using Fourier's law, one can only get the apparent thermal conductivity, which is a function of heat flux.

Cao et al. calculated the difference between apparent and intrinsic thermal conductivities of single carbon nanotube (CNT) based on the theory of thermomass (Cao and Guo, 2007). Considering a constant temperature difference of $20 \mathrm{~K}$ at two boundaries of CNT with an intrinsic thermal conductivity of $5000 \mathrm{Wm}^{-1} \mathrm{~K}^{-1}$. The heat flux along the CNT decreases as the length of CNT increases. Because of the extremely small cross sectional area of CNT, the heat flux of CNT exceeds $10^{9} \mathrm{Wm}^{-2}$ and the inertia effect of thermomass cannot be neglected. Eq. (17) predicts that the apparent thermal conductivity of CNT increases as heat flux decreases, or saying as the length of CNT increases, as shown in Fig. 3 (a).
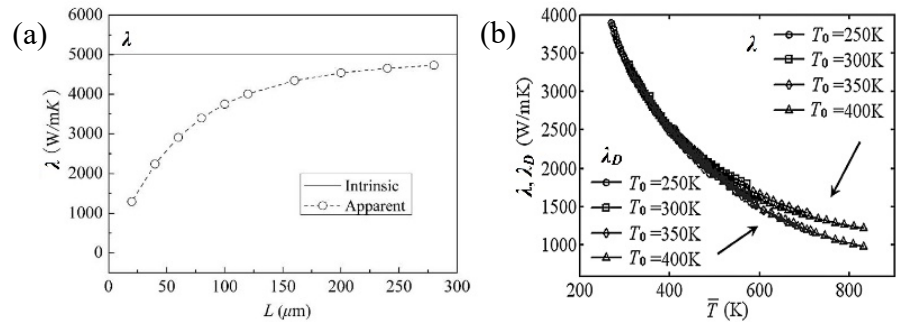

Fig. 3 Apparent and intrinsic thermal conductivities of CNT: (a) thermal conductivity changing with length, $\lambda$ and $\lambda_{D}$ are the intrinsic and apparent thermal conductivities; (b) thermal conductivity changing with temperature. Reprinted with permissions from AIP and ASME Publishing Group (Cao and Guo, 2007; Wang et al., 2010).

Figure 3 shows the difference between the apparent and intrinsic thermal conductivities of CNT, where Fig. 3 (a) is the result changing with length and Fig. 3 (b) is the result changing with temperature. The data of Fig. 3 (b) were calculated from the experimental results reported in Ref. (Pop et al., 2006). It is seen that the apparent thermal conductivity is always smaller than the intrinsic thermal conductivity. In Fig. 3 (a), the heat flux of CNT decreases as length increases at fixed boundary temperatures. In Fig. 3 (b), the thermal conductivity of CNT was measured by using a self-heating method and the high temperature of CNT corresponds to the high electrical heating power, i.e. high heat flux. Both theoretical and experimental data in Fig. 3 demonstrates that the apparent thermal conductivity of CNT approaches the intrinsic value with decreasing heat flux at longer length or at lower temperature, as predicted by Eq. (17).

\subsubsection{Size dependence of thermal conductivity of 1D nanomaterial}

Thermomass theory modeled heat conduction in solid as phonon (thermomass) gas flow in porous medium. At nanoscales, the characteristic size of nanomaterial may be comparable with the mean free path (MFP) of phonons. In this case, the continuum hypothesis may not be valid. Similar to the normal gas with scale approaching the MFP of molecules, the rarefied phonon gas effect should be taken into account. Dong et al. added a second order resistance term in the general heat conduction law to address the rarefied phonon gas effect (Dong et al., 2014). The modified resistance term of phonon gas is given as:

$$
f_{h}=-\frac{\mu}{K} u_{h}+\mu \nabla^{2} u_{h}
$$

where $\mu$ and $K$ are the fluid viscosity and permeability of porous medium, respectively. The second term is known as Brinkman term, which is usually negligible at macroscopic scales and at normal gas velocity. If the rarefied gas effect is important as the characteristic size decreases to the MFP, the normally used non-slip boundary is not accurate and a slip boundary should be used instead. In this case, the Brinkman term is no longer negligible. In order to calculate the accurate velocity profile that is different from the non-slip Poiseuille flow, an effective viscosity reduction (EVR) model was used with low computation cost. Considering the rarefied phonon gas effect by using EVR model, the effective thermal conductivity of 1D nanomaterial is shown in Fig. 4.

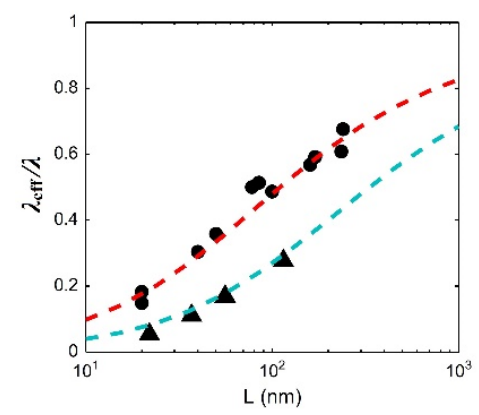

Fig. 4 Normalized thermal conductivity of 1D nanomaterial changing with length. The solid circles and triangles are the experimental data of nanofilm and nanowire, respectively. The red and blue dashed lines are the calculated results based on the modified general heat conduction law. Reprinted with permissions from Elsevier Publishing Group (Dong et al., 2014).

Fig. 4 shows that the thermal conductivities of nanofilm and nanowire increase with increasing length and approach the bulk value. The size dependent thermal conductivity can be well explained by the modified general heat conduction law, where the additional Brinkman term is used to evaluate the rarefied phonon gas effect caused by the enhanced viscous friction at the nanoscale boundaries.

\subsection{Least action principle in heat transfer}

The least action principle is one of the most important principles in physics and has been widely applied in mechanics, optics, electronics, etc. It is known that the least action principle in classical mechanics and optics can be only used in the reversible processes. For the irreversible processes in heat conduction or mass diffusion, Onsager has proposed the minimum energy dissipation principle, where the entropy generation rate, defined as the dot product of thermodynamic force and thermodynamic flow, was used as a measure of irreversibility in any linear transport processes (Onsager, 1931). Fourier's law in heat conduction can be derived through the variation of half entropy generation rate on the assumption that the thermal conductivity is proportional to the reciprocal of the square of temperature. Usually the thermal conductivity of material can be a function of temperature, but the 
specific requirement as the inverse square of temperature is not physically valid. It indicates that it is not proper to use the entropy generation rate as the measure of irreversibility in the heat transfer process.

In the theory of entransy, the dissipation rate of entransy is given as the dot product of heat flux and temperature gradient:

$$
\dot{\phi}_{g}=-q \cdot \nabla T
$$

The dissipation function of entransy is given as:

$$
\dot{\phi}_{J}=\frac{q^{2}}{2 \lambda}
$$

The variation equation of action $\dot{\phi}_{g}-\dot{\phi}_{J}$ is written as:

$$
\delta\left(\dot{\phi}_{g}-\dot{\phi}_{J}\right)=\delta\left(-q \cdot \nabla T-\frac{q^{2}}{2 \lambda}\right)=0
$$

If the thermal conductivity $\lambda$ is a constant, the calculation result of Eq. (21) is $q=-\lambda \nabla T$, which is actually the Fourier's law. It proves that the half of entransy dissipation rate is the action of heat conduction and the minimum entransy dissipation is the least action principle in heat transfer. It demonstrates that the entransy dissipation rate is really the measure of irreversibility in heat transfer process. It is also noted that in the wellknown finite element method (FEM) analysis of heat conduction, the action (or Lagrange's function) is in fact the half of entransy dissipation rate, which gives a clear physical explanation of the FEM in thermal analysis.

It is important to know that the least action principle based on entransy dissipation requires an assumption that the thermal conductivity is constant in Eq. (21). However in practical applications, the thermal conductivity of material is usually temperature dependent, which makes the constitutive relation in Eq. (21) a non-linear problem. In this case, a generalized temperature $F(T)$ should be used and the conclusions above is still valid (Chen and Pfender, 1982).

$$
F(T)=\int_{T_{0}}^{T} \frac{\lambda\left(T^{*}\right)}{\lambda_{0}\left(T_{0}\right)} \mathrm{d} T^{*}
$$

where $T_{0}$ is the reference temperature and $\lambda_{0}$ is the thermal conductivity at the reference temperature. Thus the heat conduction law with temperature-dependent thermal conductivity can be re-written as:

$$
q=-\lambda(T) \nabla T=-\lambda_{0} \nabla F
$$

It is seen that the thermal conductivity $\lambda_{0}$ is constant in Eq. (23) and the derivation and conclusions from Eq. (19) to Eq. (21) are all valid if the generalized entransy dissipation rate is used as:

$$
\dot{\phi}_{g e}=-q \cdot \nabla F=\lambda_{0}(\nabla F)^{2}
$$

The least action principle based on generalized entransy dissipation rate can be used to derive the law of heat conduction with temperature dependent thermal conductivity.

\subsection{Applications of the general heat conduction law}

In 1998, Guo has proposed a field synergy principle (FSP) to optimize the convective heat transfer by improving the synergy between temperature-gradient field and flow field (Guo et al., 1998). The energy equation in two-dimensional laminar boundary layer is given as:

$$
\rho C_{p}\left(u \frac{\partial T}{\partial x}+v \frac{\partial T}{\partial y}\right)=\frac{\partial}{\partial y}\left(\lambda \frac{\partial T}{\partial y}\right)
$$

Integrating Eq. (25) along the thickness direction of the thermal boundary layer yields:

$$
\int_{0}^{\delta_{t}} \rho C_{p}\left(u \frac{\partial T}{\partial x}+v \frac{\partial T}{\partial y}\right) \mathrm{d} y=-\left.\lambda \frac{\partial T}{\partial y}\right|_{w}
$$

where $\delta_{t}$ is the thickness of thermal boundary layer. Also we have

$$
u \frac{\partial T}{\partial x}+v \frac{\partial T}{\partial y}=\boldsymbol{U} \cdot \nabla T
$$

where $u$ and $v$ are the flow velocities in $x$ and $y$ directions, $\boldsymbol{U}$ is the velocity vector. Then the Eq. (26) can be re-written as:

$$
\int_{0}^{\delta_{t}} \rho C_{p}(\boldsymbol{U} \cdot \nabla T) \mathrm{d} y=-\left.\lambda \frac{\partial T}{\partial y}\right|_{w}
$$

The dimensionless Nusselt number can be derived as:

$$
N u_{x}=\operatorname{Re}_{x} \operatorname{Pr} \int_{0}^{1}(\overline{\boldsymbol{U}} \cdot \nabla \bar{T}) \mathrm{d} \bar{y}=\operatorname{Re}_{x} \operatorname{Pr} \int_{0}^{1}(|\overline{\boldsymbol{U}}| \cdot|\nabla \bar{T}| \cdot \cos \theta) \mathrm{d} \bar{y}
$$

where $\overline{\boldsymbol{U}}=\boldsymbol{U} / \boldsymbol{U}_{\infty}, \nabla \bar{T}=\nabla T /\left[\left(T_{\infty}-T_{w}\right) / \delta_{t}\right], \bar{y}=y / \delta, T_{\infty}>T_{w}$ and $\theta$ is the angle between the velocity vector and temperature gradient, which is called synergy angle as well. Eqs. (28) and (29) are the mathematical expression of FSP, it reveals the fact that the intensity of convective heat transfer also depends on the synergy angle between two vector of velocity and temperature gradient except for the flow velocity and the temperature difference between fluid flow and solid wall. Since the introduction of FSP, many numerical simulations and experiments have been done to prove the feasibility of FSP (Zhao and Song, 2001; Tao et al., 2002; Ma et al., 2007; Yu et al., 2018; Tao et al., 2002; Guo et al., 2005; Tao et al., 2004; Chen and Meng, 2008; Chen et al., 2008). The field synergy principle can unify the physical mechanisms of various enhancement technologies of convective heat transfer, but also develop a variety of new and efficient enhancement technologies. Chen et al. has derived a field synergy equation for the convective heat transfer, by solving which, the optimal fluid flow field can be calculated with the largest field synergy number under a given constraint condition. The largest field synergy degree can been seen as the optimization criterion for the convective heat transfer (Chen et al., 2007). In 2018, Tao and his colleagues have done a series of systematic simulations for different heat exchangers, including plain plate fin, slotted fin, composite porous materials, considering both laminar and turbulent flows, constant and variable thermosphysical properties, given heat flux and given wall temperature thermal boundary conditions (Yu et al., 2018). The results showed that the FSP and entransy dissipation extremum principle, which will be discussed in the section 3.6, are consistent in the optimization of convective heat transfer, it demonstrates that both theories have inherent consistency. FSP can be regarded as a specific instantiation of entransy theory in the convective heat transfer appearing as the synergy of flow and temperature fields.

\subsection{Applications of the general heat conduction law}

The concepts of entransy, entransy dissipation and least action principle have been introduced in the previous sections. Here, we will discuss the practical application of entransy theory in the optimization of thermal systems, which is also known as the minimum entransy dissipation-based thermal resistance (EDTR) principle (Chen et al., 2013; Guo et al., 2007). The concept of thermal resistance has been widely applied in heat transfer. However, the conventional definition of thermal resistance as the temperature difference divided by heat flux is only valid in 1D heat conduction without internal heat source. So it is difficult to expand the application range of thermal resistance in complex heat transfer problems because of arbitrariness of definition of the temperature difference. ,The concept of thermal resistance for complex heat transfer problem originates from the analogy to the electrical resistance. For a multidimensional electrical conduction with a given current, the resistance can be defined as the total dissipation rate of electrical energy divided by the square of current. In this way, the entransy dissipation-based thermal resistance can be given as:

$$
R_{g}=\frac{\dot{\phi}_{g}}{Q^{2}}
$$

where $R_{g}, \dot{\phi}_{g}$ and $Q$ are the entransy dissipation-based thermal resistance, entransy dissipation rate and heat flow rate, respectively. The concept of EDTR breaks the limitation of "1D heat conduction without internal heat source" from the traditional concept of thermal resistance 
and can be used in complex heat transfer problems. For a heat exchanger with arbitrary geometrical structures, the heat exchanger effectiveness $P$ can be expressed as:

$$
P=\frac{2}{2 R_{g}^{*}+\left(1+C^{*}\right)}
$$

where $R_{g}{ }^{*}$ and $C^{*}$ are the dimensionless EDTR (ratio of EDTR to the reciprocal of the lower heat capacity rate) and heat capacity rate ratio. It is worth mentioning that Eq. (31) does not depend on the flow arrangement of heat exchanger. Smaller EDTR always corresponds to the higher heat exchanger effectiveness, unlike the optimization method based on entropy generation that cannot give a monotonic relationship to the effectiveness $P$ (Guo et al., 2010; Liang et al., 2019).

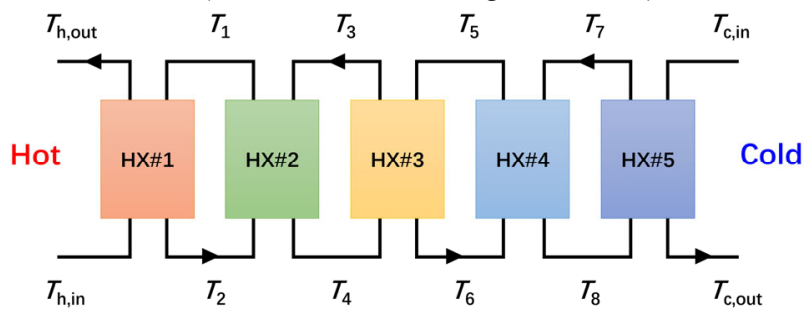

Fig. 5 Multi-loop heat exchanger network (Liang et al., 2019)

Figure 5 shows a heat exchanger network with 4 loops and 5 heat exchangers. $T_{1}$ to $T_{8}$ are the intermediate temperatures from hot fluid to cold fluid. $T_{\mathrm{h} \text {,out, }} T_{\mathrm{h} \text {,in }}, T_{\mathrm{c} \text {,out }}$ and $T_{\mathrm{c} \text {,in }}$ are the temperatures of the hot and cold fluids going out of or coming in the network. We assume that the total heat capacity flow is constant, the optimization problem is to search for the best combination of the heat capacity flow of each branch and the thermal conductance of each heat exchanger. The entransy balance equation for heat exchanger $(\mathrm{HX}) \# 1$ is:

$$
Q\left(\frac{T_{\mathrm{h}, \text { in }}+T_{\mathrm{h}, \text { out }}}{2}-\frac{T_{1}+T_{2}}{2}\right)=Q^{2} R_{g, 1}
$$

where $R_{\mathrm{g}, 1}$ is the EDTR of $\mathrm{HX \# 1:}$

$$
R_{g, 1}=\frac{1}{2}\left(\frac{1}{\dot{m}_{\mathrm{h}} c_{p, \mathrm{~h}}}-\frac{1}{\dot{m}_{1} c_{p, 1}}\right) \frac{e^{(K A)_{1}\left(\frac{1}{\dot{m}_{\mathrm{h}} c_{p, \mathrm{~h}}}-\frac{1}{\dot{m}_{1} c_{p, 1}}\right)}+1}{e^{(K A)_{1}\left(\frac{1}{\dot{m}_{\mathrm{h}} c_{p, \mathrm{~h}}}-\frac{1}{\dot{m}_{1} c_{p, 1}}\right)}-1}
$$

The entransy balance equations of HX \#2, \#3 and \#4 are:

$$
Q\left(\frac{T_{2 n-3}+T_{2 n-2}}{2}-\frac{T_{2 n-1}+T_{2 \mathrm{n}}}{2}\right)=Q^{2} R_{g, \mathrm{n}}
$$

where $R_{\mathrm{g}, \mathrm{n}}$ is the EDTR of HX\#n, $n=2,3,4$. The expression of EDTR of $\mathrm{HX} \# n$ is given as:

$$
R_{g, n}=\frac{1}{2}\left(\frac{1}{\dot{m}_{n-1} c_{p, n-1}}-\frac{1}{\dot{m}_{n} c_{p, n}}\right) \frac{e^{(K A)_{n}\left(\frac{1}{\dot{m}_{n-1} c_{p, n-1}}-\frac{1}{\dot{m}_{n} c_{p, n}}\right)}+1}{e^{(K A)_{n}\left(\frac{1}{\dot{m}_{n-1} c_{p, n-1}}-\frac{1}{\dot{m}_{n} c_{p, n}}\right)}-1}
$$

where $\dot{m}_{n}$ and $c_{p, n}$ are the mass flow rate and heat capacity at constant pressure of the \#n fluid loop. The entransy balance equation of HX\#5 is:

$$
Q\left(\frac{T_{7}+T_{8}}{2}-\frac{T_{\mathrm{c}, \text { in }}+T_{\mathrm{c}, \mathrm{out}}}{2}\right)=Q^{2} R_{g, 5}
$$

where $R_{\mathrm{g}, 5}$ is the EDTR of HX\#5, written as:

$$
R_{g, 5}=\frac{1}{2}\left(\frac{1}{\dot{m}_{4} c_{p, 4}}-\frac{1}{\dot{m}_{c} c_{p, c}}\right) \frac{e^{(K A)_{5}\left(\frac{1}{\dot{m}_{4} c_{p, 4}}-\frac{1}{\dot{m}_{c} c_{p, c}}\right)}+1}{e^{(K A)_{5}\left(\frac{1}{\dot{m}_{4} c_{p, 4}}-\frac{1}{\dot{m}_{c} c_{p, c}}\right)}-1}
$$

Summing the Eqs. (32), (34) and (36) yields:

$$
Q\left(\frac{T_{\mathrm{h}, \text { in }}+T_{\mathrm{h}, \text { out }}}{2}-\frac{T_{\mathrm{c}, \text { in }}+T_{\mathrm{c}, \text { out }}}{2}\right)=Q^{2} \sum_{n=1}^{5} R_{g, 5}
$$

The left side of Eq. (38) are the total amount of heat transferred in HX network and the boundary temperatures, the right side of Eq. (38) are the EDTR of each HX as a function of heat capacity flow and heat conductance $K A$. Hence, Eq. (38) is a "bridge" connecting the boundary conditions of a complex HX network and the internal structural parameters of each HX. It is the entransy balance equation of the whole HX network. Furthermore, Eq. (38) is independent of any specific optimization objective or known conditions, so it can be used as a general constraint equation for optimizing the HX network.

From this example, it can be seen that the optimization method based on entransy is to treat the HX network as a whole and analyze its heat transfer performance from the energy (thermomass potential energy) point of view. It is much different from the conventional optimization method that divides the whole system into different components and then builds each one's constraint equation. Eq. (38) does not involve any intermediate temperatures, thus the constraint equation is much simplified and easy to use. We would like to emphasize that the minimum entropy generation principle does not always lead to the best performance of heat exchanger, because it is not proper to use the entropy generation rate as the Lagrange's function. The entransy dissipation rate is the action or Lagrange's function in the minimum action principle. The extreme dissipation rate of entransy corresponds the best performance of heat exchanger.

\section{NEW ANALYTICAL METHODS}

\subsection{Vector method and energy method in mechanics}

As well known, Newtonian mechanics developed in the 17th century is the basis of engineering sciences, which is a typical vector method (Harrison and Nettleton, 1997). Newton's three laws of motion describes the relationship between forces and movement of a rigid body, considering vector quantities of motion, particularly accelerations, momenta, forces of a body. So Newtonian mechanics can be also named as vectorial mechanics. The vector method in Newtonian mechanics can be applied not only on rigid bodies, but also on deformable bodies and fluids. The differential equation of a moving solid or fluid can be derived based on Newtonian mechanics and predicts the movement rules of the body. The vector method in mechanics can provide extremely accurate results when the speed of object is much smaller than the speed of light, and also the size of object is not as small as atoms. After Newtonian mechanics, the analytical mechanics has been developed. Different from the vectorial mechanics, the analytical mechanics uses scalar properties to describe the motion of a body, such as kinetic energy and potential energy. So the analytical mechanics is also known as the energy method. The equations of motion can be derived by the principle about the scalar's variation, where the kinetic and potential energies of system are expressed in a generalized coordinate with constraints. Usually, the energy method to solve mechanical problems has greater efficiency than the vector method.

\subsection{Vector method in heat transfer}

In classic heat transfer, the essence of Fourier's law is actually a law between two vectors. Heat flux and temperature gradient are both vectors with directions and quantities. Fourier's law describes the constitutive 
relationship between heat flux and temperature gradient, which is same as Newton's law of viscosity in the vectorial fluid mechanics. Thermomass theory clearly indicates that the physical connotation of Fourier's law is the balance of driving force and resistant force of thermomass fluid, as discussed in section 3.1. On this basis, the thermomass momentum conservation equation is established by the vector method similar to the fluid mechanics, which is actually the general heat conduction law. The new principles and methods in thermomass theory predicts new heat transfer phenomenon, for example. non-Fourier heat conduction phenomena can occur even in steady-state cases under ultra-high heat flux conditions. In this case, the spatial thermomass inertia cannot be ignored and may cause significant deviation between the experimental result and prediction by Fourier's law. Under the ultra-fast heating conditions, if the temporal thermomass inertia cannot be ignored, the general heat conduction law will be reduced into $\mathrm{C}-\mathrm{V}$ model to describe the transient thermal wave phenomenon.

\subsection{Energy method in heat transfer}

Unlike the theory of mechanics, there is no energy method in the classic theory of heat transfer since heat has been regarded as energy. Because of the mass nature of heat during its transfer process, a new quantity, thermomass energy, has been introduced, which makes it possible to establish the energy method in heat transfer. This energy method with thermomass energy (scalar) as the core physical quantity, similar to the energy method in the analytical mechanics, can be established to analyze and optimize heat transfer problems using the variational method from a global point of view. Because the entransy is a simplified form of thermomass potential energy, the entransy dissipation is essentially the thermomass energy dissipation. So the principle of minimum entransy dissipation, which is essentially the principle of minimum energy dissipation in heat transfer, is derived based on the energy method. The least action principle is in fact derived by using the energy method as well. In addition, for multi-objective optimization of complicated heat exchanger network or thermodynamic system, the entransy (thermomass potential energy) balance equation provides a general constraint condition for their optimization.

\section{CONCLUSIONS}

1. The development of high and new technology challenges the existing heat transfer discipline in two aspects: (1) Under the extreme conditions such as ultra-fast laser heating or nanoscale heat transfer, the classical Fourier's law of heat conduction is no longer valid; (2) The principle of minimum entropy generation is not applicable to the analysis and optimization of heat transfer problem without energy conversion. Unlike the normal approaches to cope with the challenges, we start from a re-examination of the fundamental issues in heat transfer, which include the nature of heat, Fourier's law of heat conduction and the analytical method of heat transfer process, and can then introduce a series of new physical quantities, new laws and principles and establish a new analytical method.

2. According to Einstein's mass-energy relationship, the concept of thermomass is established, and then it is put forward that heat has "energy-mass" duality, that is, when heat is converted to other forms of energy, it acts as energy, and when heat is tranferred, it acts as mass. On this basis, new physical quantities such as pressure and drift velocity of thrmomass gas are introduced, and the thermomass conservation equation and the thermomass momentum conservation equation are established by the method similar to fluid mechanics. The conservation equation of thermomass momentum is actually a general law of heat conduction, which has been verified by experiments of heat conduction in a metallic thin film with ultra-high heat flux. Under different simplified conditions, the general heat conduction law will be reduced into Fourier's heat conduction law, C-V equation and steady-state non-Fourier heat conduction models, and their physical meanings are clarified respectively as well. The general heat conduction law is applicable to thermal analysis and thermal design under the extreme conditions such as ultra-fast laser heating and nanoscale heat transfer.

3. By comparing the heat conduction with the electric conduction and the fluid flow process, new physical quantities such as entransy, entransy dissipation and entransy dissipation based-thermal resistance are introduced. The physical meaning of entransy is the ability of incompressible medium to transfer heat. The traditional concept of thermal resistance can only be applied to $1 \mathrm{D}$ heat transfer without internal heat source, while the entransy dissipation-based thermal resistance can be widely applied to multi-dimensional heat transfer problems with internal heat source. The principle of minimum entransy dissipation is put forward, from which Fourier's law of heat conduction with constant thermal conductivity can be derived. Therefore, the principle of minimum entransy dissipation is the least action principle in heat transfer. The principle of minimum entransy dissipation based-thermal resistance is proposed, that is, when the entransy dissipation based-thermal resistance is minimum, the heat transfer performance is optimal. This principle can be used to optimize heat conduction, convection and radiation heat transfer processes and heat exchanger performance in order to improve their energy efficiencies.

4. The analyses show that entropy and entransy are two totally different physical quantities. The former is the state quantity of a compressible system, which is a function of two independent parameters. Its physical meaning is the unavailable part of heat output from the system to do work through a reversible thermal engine. The latter is the state quantity of an incompressible system, which is a function of temperature only, and its physical meaning is the ability of an incompressible system to transfer heat. The entropy generation is a measure of irreversibility of heat transfer process related to heat-work conversion, while the entransy dissipation is a measure of irreversibility of heat transfer process without heat-work conversion. Fourier's law of heat conduction with constant thermal conductivity can be derived based on the principle of minimum entransy dissipation, while the derived heat conduction law based on the principle of minimum entropy production requires that the thermal conductivity is inversely proportional to the square of temperature, which is inconsistent with the practical situation. In short, the entropy is the core physical quantity in thermodynamics, and the entransy is the core physical quantity in heat transfer.

5. Based on the study of physical mechanism of convective heat transfer, the principle of field synergy between the velocity vector field and temperature gradient vector field is proposed, that is, the better the synergy between fluid velocity vector field and temperature gradient vector field, the better the performance of convective heat transfer. The field synergy equation of convective heat transfer is derived by the variational method, solving which the optimal velocity field can be obtained under the given pump power condition. The field synergy principle can unify the physical mechanisms of various enhancement technologies of convective heat transfer, but also develop a variety of new and efficient enhancement technologies. Since the field synergy equation is derived under the condition of minimum entransy dissipation, and a large number of examples show that the results of heat transfer optimization based on the field synergy principle and the minimum entransy dissipation-based thermal resistance principle are the same. Hence, these two principles are mutually consistent.

6. In the classic heat transfer theory both heat conduction and convective heat transfer are based on Fourier's law of heat conduction. Fourier's law of heat conduction and general law of heat conduction are both the local laws, in which the heat flow and temperature gradient are vectors, and quantities representing heat transfer performance are obtained by solving differential equation of heat transfer, so they are similar to the vector method in Newton mechanics. Since a new energy form, thermomass energy, is introduced for the heat transfer process, an 
energy method similar to that in the analytical mechanics, i.e. energy (scalar) as the core physical quantity, can be established to analyze and optimize heat transfer problems using the variational method from a global point of view. Because the entransy is a simplified form of thermomass potential energy, the entransy dissipation is essentially the thermomass energy dissipation. So the principle of minimum entransy dissipation, which is essentially the principle of minimum energy dissipation in heat transfer, is derived based on the energy method. In addition, for multi-objective optimization of thermodynamic system, the balance equation of thermomass energy (entransy) can be used as a constraint condition to optimize the performance of the thermodynamic system, by the variational method.

7. On the basis of classic heat transfer theory, a series of new physical quantities are introduced, and then some new principles and laws of heat transfer process are put forward with the help of physical and mechanical principles, and a new analytical method are established, thus forming an independent knowledge system. This independent knowledge system may be called "Physical heat transfer" because its emphasis is on the study of heat transfer law and the analysis of its physical mechanism rather than detailed mathematical treatment.

\section{ACKNOWLEDGEMENTS}

This research was funded by National Youth 1000 Talents Program in China and National Natural Science Foundation of China grant no. 51976096.

\section{NOMENCLATURE}

$\begin{array}{ll}c & \text { Light speed }(\mathrm{m} / \mathrm{s}) \\ E & \text { Total energy }(\mathrm{J}) \\ f & \text { Resistant force }(\mathrm{N}) \\ G & \text { Entransy }(\mathrm{J} \cdot \mathrm{K}) \\ K & \text { permeability of porous medium } \\ M & \text { Mass }(\mathrm{kg}) \\ P & \text { Pressure }\left(\mathrm{N} / \mathrm{m}^{2}\right) \\ Q & \text { Heat }(\mathrm{W}) \\ R & \text { Thermal resistance }\left(\mathrm{W} / \mathrm{m}^{2} \cdot \mathrm{K}\right) \\ S & \text { Entropy }(\mathrm{J} / \mathrm{K}) \\ T & \text { Temperature }(\mathrm{K}) \\ u & \text { Velocity }(\mathrm{m} / \mathrm{s}) \\ x & \text { coordinate }(\mathrm{m})\end{array}$

\section{Greek Symbols}

$\begin{array}{ll}\gamma & \text { Grüneisen constant } \\ \lambda & \text { Thermal conductivity }(\mathrm{W} / \mathrm{m} \cdot \mathrm{K}) \\ \rho & \text { Density }\left(\mathrm{kg} / \mathrm{m}^{3}\right)\end{array}$

\section{Subscripts}

$h$

Thermal mass

\section{REFERENCES}

Lienhard, J. H., 2019, “A Heat Transfer Textbook," 5th edition, Dover Civil and Mechanical Engineering, Dover Publications, USA.

Kroes, P., Bakker, M., 2013, “Technological Development and Science in the Industrial Age: New Perspectives on the Science-Technology Relationship," Springer Science \& Business Media, Germany.

Look Jr., D. C., et al., 1988, "Engineering Thermodynamics," Van Nostrand Reinhold (International) Co. Ltd, UK.

Reynolds, A. J., 1971, "Thermo-Fluid Dynamics," Wiley-Interscience, London.

Fuchs, H. U., 2010, "The Dynamics of Heat, A Unified Approach to
Thermodynamics and Heat Transfer", 2nd edition, Springer Co. Ltd, USA.

Wang, H. D., Cao, B. Y., Guo, Z. Y., 2012, "Non-Fourier Heat Conduction in Carbon Nanotubes," J. Heat Transfer, 134, 051004. https://doi.org/10.1115/1.4005634

Bishri, A. H., 1999, "Modelling Non-Fourier Heat Conduction with Periodic Thermal Oscillation Using the Finite Integral Transform," Appl. Math. Model., 23, 899-914. https://doi.org/10.1016/S0307904X(99)00017-7

Herwig, Beckert, H., K., 2000, "Fourier Versus Non-Fourier Heat Conduction in Materials with A Nonhomogeneous Inner Structure," $J$. Heat Transfer, 122, 363-365. https://doi.org/10.1115/1.521471

Zhou, J. H., Zhang, Y. W., Chen, J. K., 2008, "Non-Fourier Heat Conduction Effect on Laser-Induced Thermal Damage in Biological Tissues," Numer. Heat Transfer A: Appl., 54, 1-19. https://doi.org/10.1080/10407780802025911

Mohajer, M., Ayani, M. B., Tabrizi, H. B., 2016, "Numerical Study of Non-Fourier Heat Conduction in A Biolayer Spherical Living Tissue During Hyperthermia," J. Therm. Biol., 62, 181-188. http://doi.org/10.1016/j.jtherbio.2016.06.019

Guo, Z. Y., Xu, Y. S., 1992, "Non-Fourier Heat Conduction in IC Chip," Proceedings of Intersociety Conference on Thermal Phenomena in Electronic Systems, Austin, USA, Feb. 5-8.

Shiomi, J., Maruyama, S., 2006, "Non-Fourier Heat Conduction in A Single-Walled Carbon Nanotube: Classical Molecular Dynamics Simulations," Phys. Rev. B, 73, 205420. https://doi.org/10.1103/PhysRevB.73.205420

Zhang, D. M., Li, L., Li, Z. H., et al., 2006, "Non-Fourier Heat Conduction Studying on High-Power Short-Pulse Laser Ablation Considering Heat Source Effect," Eur. Phys. J. Appl. Phys., 33, 91-96. https://doi.org/10.1051/epjap:2006007

Bertola, V., Cafaro, E., 2008, "A Critical Analysis of the Minimum Entropy Production Theorem and Its Application to Heat and Fluid Flow," Int. J. Heat Mass Transfer, 51, 1907-1912. https://doi.org/10.1016/j.ijheatmasstransfer.2007.06.041

Kjelstrup, S., Bedeaux, D., Sauar, E., 2000, "Minimum Entropy Production by Equipartition of Forces in Irreversible Thermodynamics," Ind. Eng. Chem. Res. 39, 4434-4436. https://doi.org/10.1021/ie0006920

Prigogine, I., 1967, "Introduction to Thermodynamics of Irreversible Processes," 3rd ed., Wiley, New York.

Bejan, A., 1977, "The Concept of Irreversibility in Heat Exchanger Design: Counterflow Heat Exchangers for Gas-To-Gas Applications," $J$. Heat Transfer - Trans. ASME, 99, 374-380. http://doi.org/10.1115/1.3450705

Bejan, A., 1979, "Study of Entropy Generation in Fundamental Convective Heat Transfer," J. Heat Transfer - Trans. ASME, 101, 718725. https://doi.org/10.1115/1.3451063

Bejan, A., 1982, "Entropy Generation through Heat and Fluid Flow," John Wiley \& Sons, New York.

Bejan, A., 1996, "Entropy Generation Minimization: The New Thermodynamics of Finite-Size Devices and Finite-Time Processes," J. Appl. Phys. 79, 1191-1218. https://doi.org/10.1063/1.362674

Bejan, A., 1996, "Entropy Generation Minimization: The Method of Thermodynamic Optimization of Finite-Size Systems and Finite-Time Processes," $\boldsymbol{C R C}$.

Bejan, A., 2002, "Fundamentals of Exergy Analysis, Entropy Generation 
Minimization, and The Generation of Flow Architecture," Int. J. Energy Res., 26, 545-565. https://doi.org/10.1002/er.804

Hesselgreaves, J. E., 2000, "Rationalisation of Second Law Analysis of Heat Exchangers," Int. J. Heat Mass Transfer 43, 4189-4204. https://doi.org/10.1016/S0017-9310(99)00364-6

Pandey, S. D., Nema, V. K., 2011, “An Experimental Investigation of Exergy Loss Reduction in Corrugated Plate Heat Exchanger," Energy 36, 2997-3001. https://doi.org/10.1016/j.energy.2011.02.043

Sekulic, D. P., 1986, "Entropy Generation in A Heat Exchanger," Heat Transfer Eng. 7, 83-88. https://doi.org/10.1080/01457638608939647

Tzou, D. Y., 1996, "Microscale Heat Transfer, The Lagging Behavior," Taylor \& Francis Co. Ltd, USA.

Rukolaine, S. A., 2014, "Unphysical Effects of the Dual-Phase-Lag Model of Heat Conduction," Int. J. Heat Mass Transfer, 78, 58-63. https://doi.org/10.1016/j.ijheatmasstransfer.2014.06.066

Chen, J. K., Tzou, D. Y., Beraun, J. E., 2006, “A Semiclassical TwoTemperature Model for Ultrafast Laser Heating," Int. J. Heat Mass Transfer, 49, 307-316. https://doi.org/10.1016/j.ijheatmasstransfer.2005.06.022

Cattaneo, C., 1948, "Sulla conduzione del calore," Atti Sem. Mat. Fis. Univ. Modena, 3, 83-101.

Vernotte, P., 1958, "Paradoxes in the Continuous Theory of the Heat Equation," C. R. Acad. Sci., 246, 3154-3155.

Wang, H. D., 2014, "Theoretical and Experimental Studies on NonFourier Heat Conduction Based on Thermomass Theory," Springer Co. $\boldsymbol{L t d}$, USA.

Wang, M. R., Cao, B. Y., Guo, Z. Y., 2010, "General Heat Conduction Equations Based on the Thermomass Theory," Frontiers Heat Mass Transfer, 1, 013004. https://doi.org/10.5098/hmt.v1.1.3004

Cao, B. Y., Guo, Z. Y., 2007, "Equation of Motion of a Phonon Gas and Non-Fourier Heat Conduction," J. Appl. Phys., 102, 5, 053503. https://doi.org/10.1063/1.2775215

Guo, Z. Y., Hou, Q. W., 2010, "Thermal Wave Based on the Thermomass Model," J. Heat Transfer ASME, 132, 072403. https://doi.org/10.1115/1.4000987

Dong, Y., Guo, Z. Y., 2011, "Entropy Analyses for Hyperbolic Heat Conduction Based on the Thermomass Model," Int. J. Heat Mass Transfer, 54,

1924-1929. https://doi.org/10.1016/j.ijheatmasstransfer.2011.01.011

Dong, Y., Cao, B. Y., Guo, Z. Y., 2017, "Thermomass Theory: A Mechanical Pathway to Analyze Anomalous Heat Conduction in Nanomaterials," Nanomechanics, Alexander Vakhrushev, IntechOpen.

Dong, Y., Cao, B. Y., Guo, Z. Y., 2012, “General Expression for Entropy Production in Transport Processes Based on the Thermomass Model," Phys. Rev. E., 85, 061107. https://doi.org/10.1103/PhysRevE.85.061107

Gupta, M., Mukhopadhyay, S., 2019, "A Study on Generalized Thermoelasticity Theory Based on Non-Local Heat Conduction Model with Dual-Phase-Lag," J. Therm. Stress., 42, 1123-1135. https://doi.org/10.1080/01495739.2019.1614503

Freitas, R. S. M., Stampa, C. S., Lobão, D. C., et al., 2016, "Numerical study concerning thermal responses of nanofilms under the thermomass theory," Therm. Eng., 15, 57-66. http://dx.doi.org/10.5380/reterm.v15i1.62148

Wang, Y. Z., Zhang, X. B., Song, X. N., 2014, "A Generalized Theory of Thermoelasticity Based on Thermomass and Its Uniqueness Theorem," Acta Mechanica, 225, 797-808. http://dx.doi.org/10.1007/s00707-013- $\underline{1001-4}$

Jou, D., Sellitto, A., Alvarez, F. X., 2011, "Heat Waves and Phonon-Wall Collisions in Nanowires," Proc. R. Soc. A, 467, 2520-2533. http://dx.doi.org/10.1098/rspa.2010.0645

Cimmelli, V. A., Rogolino, P., Sellitto, A., 2016, “A Nonlinear Model of Thermoelectricity with Two Temperatures: Application to Quasicrystalline Nanowires," J. Math. Phys., 57, 043507. https://doi.org/10.1063/1.4947060

Dong, Y., 2016, "Thermal Rectification Based on Phonon Hydrodynamics and Thermomass Theory," Commun. Appl. Ind. Math., 7, 26-38. http://dx.doi.org/10.1515/caim-2016-0004

Chen, Q., Liang, X. G., Guo, Z. Y., 2013, "Entransy Theory for The Optimization of Heat Transfer - A Review and Update," Int. J. Heat Mass Transfer, 63, 65-81.

http://dx.doi.org/10.1016/j.ijheatmasstransfer.2013.03.019

Cheng, X. T., Zhang, Q. Z., Liang, X. G., 2012, “Analyses of Entransy Dissipation, Entropy Generation and Entransy-Dissipation-Based Thermal Resistance on Heat Exchanger Optimization," Appl. Therm. Eng. 38, 31-39. http://dx.doi.org/10.1016/j.applthermaleng.2012.01.017

Wang, H. R., Liu, Z. Y., Wu, H. Y., 2017, "Entransy Dissipation-Based Thermal Resistance Optimization of Slab LHTES System with Multiple Pcms Arranged in A 2D Array," Energy, 138, 739-751. http://dx.doi.org/10.1016/j.energy.2017.07.089

Guo, Z. Y., Cheng, X. G., Xia, Z. Z., 2003, “Least Dissipation Principle of Heat Transport Potential Capacity and Its Application in Heat Conduction Optimization," Chin. Sci. Bull., 48, 406-410. http://dx.doi.org/10.1360/03tb9085

Meng, J. A., Liang, X. G., Li, Z. X., 2005, "Field Synergy Optimization and Enhanced Heat Transfer by Multi-Longitudinal Vortexes Flow in Tube," Int. J. Heat Mass Transfer, 48, 3331-3337. https://doi.org/10.1016/j.ijheatmasstransfer.2005.02.035

Chen, L. G., Wei, S. H., Sun, F. R., 2008, "Constructal Entransy Dissipation Minimization for 'Volume-Point' Heat Conduction," J. Phys. $D$ - Appl. Phys., 41, 195506. https://doi.org/10.1088/0022$\underline{3727 / 41 / 19 / 195506}$

Chen, L. G., Wei, S. H., Sun, F. R., 2011, "Constructal Entransy Dissipation Rate Minimization of A Disc," Int. J. Heat Mass Transfer, 54, 210-216. https://doi.org/10.1016/j.ijheatmasstransfer.2010.09.050

Chen, Q., Guo, Z. Y., 2010, "Entransy Theory and Its Application to Heat Transfer Analyses in Porous Media," Int. J. Nonlinear Sci. Numer. Simul., 11, 11-22. https://doi.org/10.1515/IJNSNS.2010.11.1.11

Chen, Q., Wang, M. R., Pan, N., et al., 2009, "Irreversibility of Heat Conduction in Complex Multiphase Systems and Its Application to The Effective Thermal Conductivity of Porous Media," Int. J. Nonlinear Sci. Numer. Simul., 10, 57-66. https://doi.org/10.1515/IJNSNS.2009.10.1.57

Li, Q. Y., Chen, Q., 2012, “Application of Entransy Theory in The Heat Transfer Optimization of Flat-Plate Solar Collectors," Chin. Sci. Bull., 57, 299-306. http://dx.doi.org/10.1007/s11434-011-4811-6

Song, B., Guo, Z. Y., 2011, "Robustness in The Volume-To-Point Heat Conduction Optimization Problem," Int. J. Heat Mass Transfer, 54, 4531-4539. https://doi.org/10.1016/j.ijheatmasstransfer.2011.06.025

Chen, Q., Ren, J. X., Meng, J. A., 2007, "Field Synergy Equation for Turbulent Heat Transfer and Its Application," Int. J. Heat Mass Transfer, 50, 5334-5339. https://doi.org/10.1016/j.ijheatmasstransfer.2007.10.001

Chen, Q., Ren, J. X., 2008, "Generalized Thermal Resistance for Convective Heat Transfer and Its Relation to Entransy Dissipation," Chin. Sci. Bull., 53, 3753-3761. http://dx.doi.org/10.1007/s11434-008-0526-8 
Yuan, F., Chen, Q., 2011, "Two Energy Conservation Principles in Convective Heat Transfer Optimization," Energy, 36, 5476-5485. http://dx.doi.org/10.1016/j.energy.2011.07.033

Jia, H., Liu, W., Liu, Z. C., 2012, "Enhancing Convective Heat Transfer Based on Minimum Power Consumption Principle," Chem. Eng. Sci., 69, 225-230. http://dx.doi.org/10.1016/j.ces.2011.10.044

Cheng, X. T., Liang, X. G., 2011, "Entransy Flux of Thermal Radiation and Its Application to Enclosures with Opaque Surfaces," Int. J. Heat Mass Transfer, 54, 269-278. https://doi.org/10.1016/j.ijheatmasstransfer.2010.08.026

Cheng, X. T., Xu, X. H., Liang, X. G., 2011, "Radiative Entransy Flux in Enclosures with Non-Isothermal or Non-Grey, Opaque, Diffuse Surfaces and Its Application," Sci. China - Technol. Sci., 54, 2446-2456. http://dx.doi.org/10.1007/s11431-011-4419-8

Liu, X. B., Meng, J. A., Guo, Z. Y., 2009, "Entropy generation extremum and entransy dissipation extremum for heat exchanger optimization," Chin. Sci. Bull., 54, 943-947. https://doi.org/10.1007/s11434-009-0130$\underline{6}$

Xia, L., Feng, Y. L., Sun, X. Y., et al., 2017, "A Novel Method Based on Entransy Theory for Setting Energy Targets of Heat Exchanger Network," Chin. J. Chem. Eng. 25, 1037-1042. https://doi.org/10.1016/j.cjche.2017.03.015

Guo, Z. Y., 2018, "Energy-mass duality of heat and its applications," ES Energy Environment 1, 4-15. http://dx.doi.org/10.30919/esee8c146

Einstein, A., 1988, "Relativity: the special and the general theory, a clear explanation that anyone can understand," Wings Books, New Ed edition.

Dong, Y., Cao, B. Y., Guo, Z. Y., 2011, "Generalized Heat Conduction Laws Based on Thermomass Theory and Phonon Hydrodynamics," J. Appl. Phys. 110, 063504. https://doi.org/10.1063/1.3634113

Dong, Y., 2015, "Dynamical Analysis of Non-Fourier Heat Conduction and Its Application in Nanosystems," Springer Co. Ltd, USA.

Mares, J. J., Hubik, P., Sestak, J. et al. 2008, "Phenomenological Approach to The Caloric Theory of Heat," Thermochimica Acta, 474, 1624. https://doi.org/10.1016/j.tca.2008.05.001

Fox, R., 1971, "The Caloric Theory of Gases," Clarendon Press, Oxford, UK.

Wang, H. D., Guo, Z. Y., 2010, "Thermon Gas as The Thermal Energy Carrier in Gas and Metals," Chin. Sci. Bull. 55, 3350-3355. https://doi.org/10.1007/s11434-010-4036-0

Xu, M. T., 2011, "The Thermodynamic Basis of Entransy and Entransy Dissipation," Energy, 36, 4272-4277. http://dx.doi.org/10.1016/j.energy.2011.04.016

Guo, Z. Y., Zhu, H. Y., Liang, X. G., 2007, "Entransy - A Physical Quantity Describing Heat Transfer Ability," Int. J. Heat Mass Transfer, 50, 2545-2556. https://doi.org/10.1016/j.ijheatmasstransfer. 2006.11.034

Cheng, X. T., Liang, X. G., Guo, Z. Y., 2011, "Entransy decrease principle of heat transfer in an isolated system," Chin. Sci. Bull. 56, 847854. https://doi.org/10.1007/s11434-010-4328-4

Chen, Q., Zhu, H.Y., Pan, N., et al., 2011, “An Alternative Criterion in Heat Transfer Optimization," Proc. R. Soc. A - Math. Phys. Eng. Sci. 467, 1012-1028. https://doi.org/10.1098/rspa.2010.0293

Chen, Q., Wang, M. R., Pan, N., et al., 2009, “Optimization Principles for Convective Heat Transfer," Energy 34, 1199-1206. https://doi.org/10.1016/j.energy.2009.04.034

Maillet, D., 2019, "A Review of the Models Using the Cattaneo and Vernotte Hyperbolic Heat Equation and Their Experimental Validation," $\begin{array}{llll}\text { Int. } & \text { J. Therm. } & \text { Sci. } & \text { 139, }\end{array}$ https://doi.org/10.1016/j.ijthermalsci.2019.02.021

Wang, H. D., Liu, J. H., Guo, Z. Y., et al., 2012, "Non-Fourier Heat Conduction Study for Steady States in Metallic Nanofilms," Chin. Sci. Bull. 57, 3239-3243. https://doi.org/10.1007/s11434-012-5288-7

Wang, H. D., Cao, B. Y., Guo, Z. Y., 2010, "Heat Flow Choking in Carbon Nanotubes," Int. J. Heat Mass Transfer, 53, 21796-1800. https://doi.org/10.1016/j.ijheatmasstransfer.2010.01.010

Pop, E., Mann, D., Wang, Q., et al., 2006, "Thermal Conductance of an Individual Single-Wall Carbon Nanotube Above Room Temperature," Nano Lett. 6, 96-100. https://doi.org/10.1021/n1052145f

Dong, Y., Cao, B. Y., Guo, Z. Y., 2014, "Size Dependent Thermal Conductivity of Si Nanosystems Based on Phonon Gas Dynamics," Physica E, 56, 256-262. https://doi.org/10.1016/j.physe.2013.10.006

Onsager, L., 1931, "Reciprocal Relations in Irreversible Process. I," Phys. Rev. 37, 405-426. https://doi.org/10.1103/PhysRev.37.405

Onsager, L., 1931, "Reciprocal Relations in Irreversible Process II.," Phys. Rev. 38, 2265-2279. https://doi.org/10.1103/PhysRev.38.2265

Chen, X., Pfender, E., 1982, "Heat Transfer to A Single Particle Exposed to A Thermal Plasma," Plasma Chem. Plasma Process. 2, 185-212. https://doi.org/10.1007/BF00633133

Guo, Z. Y., Li, D. Y., Wang, B. X., 1998, “A Novel Concept for Convective Heat Transfer Enhancement," Int. J. Heat Mass Transf. 41, 2221-2225. https://doi.org/10.1016/S0017-9310(97)00272-X

Zhao, T. S., Song, Y. J., 2001, "Forced Convection in A Porous Medium Heated by A Permeable Wall Perpendicular to Flow Direction: Analyses and Measurements," Int. J. Heat Mass Transf. 44, 1031-1037. https://doi.org/10.1016/S0017-9310(00)00171-X

Tao, W. Q., He, Y. L., Wang, Q. W., et al., 2002, “A Unified Analysis on Enhancing Single Phase Convective Heat Transfer with Field Synergy Principle," Int. J. Heat Mass Transf. 45, 4871-4879. https://doi.org/10.1016/S0017-9310(02)00173-4

Ma, L. D., Li, Z. Y., Tao, W. Q., 2007, "Experimental Verification of the Field Synergy Principle," Int. Commun. Heat Mass 34, 269-276. https://doi.org/10.1016/j.icheatmasstransfer.2006.11.008

Yu, Z. Q., Wang, P., Zhou, W. J., et al. 2018, "Study on the Consistency Between Field Synergy Principle and Entransy Dissipation Extremum Principle," Int. J. Heat Mass Transf. 116, 621-634. https://doi.org/10.1016/j.ijheatmasstransfer.2017.09.044

Tao, W. Q., Guo, Z. Y., Wang, B. X., 2002, “Field Synergy Principle for Enhancing Convective Heat Transfer-Its Extension and Numerical Verifications," Int. J. Heat Mass Transfer 45, 3849-3856. https://doi.org/10.1016/S0017-9310(02)00097-2

Guo, Z. Y., Tao, W. Q., Shah, R. K., 2005, "The Field Synergy (Coordination) Principle and Its Applications in Enhancing Single Phase Convective Heat Transfer," Int. J. Heat Mass Transfer, 48, 1797-1807. https://doi.org/10.1016/j.ijheatmasstransfer.2004.11.007

Tao, W. Q., He, Y. L., Qu, Z. G., et al., 2004, “Applications of The Field Synergy Principle in Developing New Type Heat Transfer Enhanced Surfaces," J. Enhance. Heat Transfer, 11 (4), 435-451. https://doi.org/10.1615/JEnhHeatTransf.v11.i4.210

Chen, Q., Meng, J. A., 2008, "Field Synergy Analysis and Optimization of the Convective Mass Transfer in Photocatalytic Oxidation Reactors," Int. J. Heat Mass Transfer, 51, 2863-2870. https://doi.org/10.1016/j.ijheatmasstransfer.2007.09.024

Chen, Q., Ren, J. X., Guo, Z. Y., 2008, "Field Synergy Analysis and 
Optimization of Decontamination Ventilation Designs," Int. J. Heat Mass Transfer, 51, 873-881. https://doi.org/10.1016/j.ijheatmasstransfer. $\underline{2007.04 .004}$

Guo, Z. Y., Liu, X., Tao, W. Q., et al. 2010, "Effectiveness - Thermal Resistance Method for Heat Exchanger Design and Analysis," Int. J. Heat Mass Transf. 53, 5877-2884. https://doi.org/10.1016/j.ijheatmasstransfer. 2010.02.008
Liang, X. G., Chen, Q., Guo, Z. Y., 2019, "Entransy Theory for Heat Transfer Analysis and Optimizations (In Chinese)," Science Press, Beijing, China.

Harrison, H. R., Nettleton, T., 1997, "Newtonian Mechanics," Advanced Engineering, Dynamics, 1-20. 